\title{
NONCOMMUTATIVE CATALAN NUMBERS
}

\author{
ARKADY BERENSTEIN AND VLADIMIR RETAKH \\ To George Andrews on the occasion of his 80th birthday
}

\begin{abstract}
The goal of this paper is to introduce and study noncommutative Catalan numbers $C_{n}$ which belong to the free Laurent polynomial algebra $\mathcal{L}_{n}$ in $n$ generators. Our noncommutative numbers admit interesting (commutative and noncommutative) specializations, one of them related to Garsia-Haiman $(q, t)$ versions, another - to solving noncommutative quadratic equations. We also establish total positivity of the corresponding (noncommutative) Hankel matrices $H_{n}$ and introduce accompanying noncommutative binomial coefficients $\left.\left(\begin{array}{l}n \\ k\end{array}\right) \in \mathcal{L}_{n+k-1},\left(\begin{array}{l}n \\ k\end{array}\right)\right)^{\prime} \in \mathcal{L}_{n}$.
\end{abstract}

\section{Contents}

1. Introduction

1.1. Acknowledgments

2. Notation and main results

3. Proofs of main results

3.1. Proof of Propositions 2.3, 2.9, 2.20 and Theorems 2.22, 2.26

3.2. Proof of Theorems 2.32 and 2.34

3.3. Proof of Theorems 2.38 and 2.43

3.4. Proof of Theorems 2.29, 2.36

References

\section{INTRODUCTION}

Catalan numbers $c_{n}=\frac{1}{n+1}\left(\begin{array}{c}2 n \\ n\end{array}\right), n \geq 0$ are important combinatorial objects which satisfy a number of remarkable properties such as:

- The recursion $c_{n+1}=\sum_{k=0}^{n} c_{k} c_{n-k}$ for all $n \geq 0$ (with $\left.c_{0}=c_{1}=1\right)$.

- the determinantal identities $\operatorname{det}\left(\begin{array}{cccc}c_{m} & c_{m+1} & \ldots & c_{m+n} \\ c_{m+1} & c_{m+2} & \ldots & c_{m+n+1} \\ & & \ldots & \\ c_{m+n} & c_{m+n+1} & \ldots & c_{m+2 n}\end{array}\right)=1$ for $n \geq 0, m \in\{0,1\}$.

Catalan numbers admit various $q$-deformations $([2,9,16])$ and $(q, t)$-deformations $([10,11,16])$.

In this paper we introduce and study noncommutative Catalan numbers $C_{n}, n \geq 1$ which are totally noncommutative Laurent polynomials in $n$ variables and satisfy analogues of the recursion and the determinantal identities (Proposition 2.3 and equation (2.8)). It turns out that specializing these variables to appropriate powers of $q$, we recover Garsia-Haiman $(q, 1)$-Catalan numbers. Catalan numbers also satisfy a combinatorial identity (formula (4.9) in [6]) involving their truncated counterparts $c_{n}^{k}=\left(\begin{array}{c}n+k \\ k\end{array}\right)-\left(\begin{array}{c}n+k \\ k-1\end{array}\right)$ (so that $\left.c_{n}=c_{n}^{n}=c_{n}^{n-1}\right)$ :

$$
c_{n}=\sum_{\substack{a, b \in \mathbb{Z}_{\geq 0}: \\ a+b \leq n, a-b=d}} c_{n-b}^{a} c_{n-a}^{b}
$$

This work was partially supported by the NSF grant DMS-1403527 (A. B.). 
for each $n \in \mathbb{Z}_{\geq 0}$ and each $d \in \mathbb{Z}$ with $|d| \leq n$ (e.g., the right hand side does not depend on $d$ ). A $q$-deformation of $c_{n}^{k}$ was discussed in [7] under the name of $q$-ballot numbers.

We introduce noncommutative analogues of truncated Catalan numbers and establish a noncommutative version of (1.1) (Theorem 2.22). It is curious that the $c_{n}^{k}$ satisfy three more combinatorial identities, two of which involve binomial coefficients:

$$
c_{n+1}^{k}=\sum_{j=0}^{k} c_{j} c_{n-j}^{k-j}, \sum_{j=0}^{k}(-1)^{j} c_{n+k-j}^{j} \cdot\left(\begin{array}{l}
n-j \\
k-j
\end{array}\right)=0, c_{m+n}^{k}=\sum_{\ell=0}^{n} c_{m+\ell}^{k-\ell} \cdot\left(\begin{array}{c}
n \\
\ell
\end{array}\right),
$$

where $0 \leq k<n$ in the first two identities and $0 \leq k \leq m+n$ in the third one.

We establish a noncommutative generalization of the first identity (1.2) (Proposition 2.20(c)), define appropriate noncommutative versions $\left(\begin{array}{l}n \\ k\end{array}\right)$ and $\left(\begin{array}{l}n \\ k\end{array}\right)$ of binomial coefficients and establish analogues of the last two identities (1.2) with these coefficients (Corollary 2.33 and Theorem 2.34) as well as an analogue of the multiplication law for both kinds of noncommutative binomial coefficients (Theorem 2.32).

In fact, these constructions and results extend our previous work on Noncommutative Laurent Phenomenon ([3, 4]) and we expect more such Phenomena to emerge in Combinatorics, Representation Theory, Topology and related fields.

The paper is organized as follows: Section 2 contains notation and main results and the proofs are given in Section 3 .

1.1. Acknowledgments. This work was partly done during our visits to Max-Planck-Institut für Mathematik and Institut des Hautes Études Scientifiques. We gratefully acknowledge the support of these institutions. We thank Philippe Di Francesco and Rinat Kedem for their comments on the first version of the paper, particularly for explaining to us a relationship between noncommutative Stieltjes continued fractions and our noncommutative Catalan series (see Remark 2.6).

\section{Notation AND MAin RESUlts}

Let $F$ be the free group generated by $x_{k}, k \in \mathbb{Z}_{\geq 0}$ and $F_{m}$ be the (free) subgroup of $F$ generated by $x_{0}, \ldots, x_{m}$.

Denote by $\tilde{\mathcal{P}}_{n}$ the set of all monotonic lattice paths in $[0, n] \times[0, n]$ from $(0,0)$ to $(n, n)$. Clearly, $\left|\tilde{\mathcal{P}}_{n}\right|=\left(\begin{array}{c}2 n \\ n\end{array}\right)$. We say that $P \in \tilde{\mathcal{P}}_{n}$ is Catalan if for each point $p=\left(p_{1}, p_{2}\right) \in P$ one has $c(p) \geq 0$, where $c\left(p_{1}, p_{2}\right):=p_{1}-p_{2}$ is the content of $p$. Denote by $\mathcal{P}_{n} \subset \tilde{\mathcal{P}}_{n}$ the set of all Catalan paths in $[0, n] \times[0, n]$. Clearly, $\left|\mathcal{P}_{n}\right|=\frac{1}{n+1}\left(\begin{array}{c}2 n \\ n\end{array}\right)$ is the $n$-th Catalan number, which justifies the terminology.

We say that a point $p=\left(p_{1}, p_{2}\right)$ of $P \in \tilde{\mathcal{P}}_{n}$ is a southeast (resp. northwest) corner of $P$ if $\left(p_{1}-1, p_{2}\right) \in P$ and $\left(p_{1}, p_{2}+1\right) \in P$ (resp. $\left(p_{1}, p_{2}-1\right) \in P$ and $\left.\left(p_{1}+1, p_{2}\right) \in P\right)$.

To each $P \in \mathcal{P}_{n}$ we assign an element $M_{P} \in F_{n}$ by

$$
M_{P}=\vec{\prod} x_{c(p)}^{\operatorname{sgn}(p)}
$$

where the product is over all corners $p \in P$ (taken in the natural order) and $\operatorname{sgn}(p)=\left\{\begin{array}{ll}1 & \text { if } p \text { is southeast } \\ -1 & \text { if } p \text { is northwest }\end{array}\right.$.

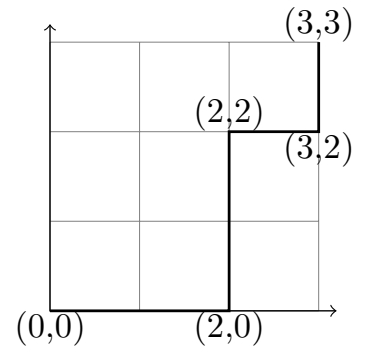

Figure 1. $M_{P}=x_{2} x_{0}^{-1} x_{1}$ for the above path $P \in \mathcal{P}_{3}$ 
We define the noncommutative Catalan number $C_{n} \in \mathbb{Z} F_{n}$ by

$$
C_{n}=\sum_{P \in \mathcal{P}_{n}} M_{P}
$$

Clearly, under the counit homomorphism $\varepsilon: \mathbb{Z} F \rightarrow \mathbb{Z}\left(x_{k} \mapsto 1\right)$ the image $\varepsilon\left(C_{n}\right)$ is $\left|\mathcal{P}_{n}\right|$, the ordinary Catalan number.

Noncommutative Catalan numbers exhibit some symmetries, the first of which is an anti-automorphism of $\mathbb{Z} F$ such that $\bar{x}_{k}=x_{k}$ for $k \in \mathbb{Z}_{\geq 0}$.

Proposition 2.1. $\bar{C}_{n}=C_{n}$ for all $n \geq 0$.

Proof. Define an involution $s_{n}: \mathbb{Z}^{2} \rightarrow \mathbb{Z}^{2}$ by $s_{n}(x, y)=(n-y, n-x)$. Clearly, $s_{n}\left(\mathcal{P}_{n}\right)=\mathcal{P}_{n}$. It is easy to see that

$$
\bar{M}_{P}=M_{s_{n}(P)}
$$

for all $P \in \mathcal{P}_{n}$. Therefore, $\bar{C}_{n}=\sum_{P \in \mathcal{P}_{n}} \bar{M}_{P}=\sum_{P \in \mathcal{P}_{n}} M_{s_{n}(P)}=\sum_{P \in \mathcal{P}_{n}} M_{P}=C_{n}$ for all $n \geq 0$.

The proposition is proved.

Example 2.2. $C_{0}=x_{0}, C_{1}=x_{1}, C_{2}=x_{2}+x_{1} x_{0}^{-1} x_{1}$,

$$
\begin{gathered}
C_{3}=x_{3}+x_{2} x_{1}^{-1} x_{2}+x_{2} x_{0}^{-1} x_{1}+x_{1} x_{0}^{-1} x_{2}+x_{1} x_{0}^{-1} x_{1} x_{0}^{-1} x_{1} \\
C_{4}=x_{4}+x_{3} x_{2}^{-1} x_{3}+x_{2} x_{0}^{-1} x_{2}+x_{3} x_{1}^{-1} x_{2}+x_{2} x_{1}^{-1} x_{3}+x_{3} x_{0}^{-1} x_{1}+x_{1} x_{0}^{-1} x_{3}+x_{2} x_{1}^{-1} x_{2} x_{1}^{-1} x_{2} \\
+x_{1} x_{0}^{-1} x_{2} x_{0}^{-1} x_{1}+x_{2} x_{1}^{-1} x_{2} x_{0}^{-1} x_{1}+x_{1} x_{0}^{-1} x_{2} x_{1}^{-1} x_{2}+x_{2} x_{0}^{-1} x_{1} x_{0}^{-1} x_{1}+x_{1} x_{0}^{-1} x_{1} x_{0}^{-1} x_{2}+x_{1} x_{0}^{-1} x_{1} x_{0}^{-1} x_{1} x_{0}^{-1} x_{1} .
\end{gathered}
$$

It turns out that our noncommutative Catalan numbers satisfy the following generalization of the wellknown classical recursion, which we prove in Section 3.1

Proposition 2.3. For $n \geq 0$ one has

$$
C_{n+1}=\sum_{k=0}^{n} C_{k} x_{0}^{-1} T\left(C_{n-k}\right), C_{n+1}=\sum_{k=0}^{n} T\left(C_{k}\right) x_{0}^{-1} C_{n-k}
$$

for all $n \in \mathbb{Z}_{\geq 0}$, where $T: \mathbb{Z} F \rightarrow \mathbb{Z} F$ is an endomorphism of $\mathbb{Z} F$ given by $T\left(x_{k}\right)=x_{k+1}$ for all $k \in \mathbb{Z} \geq 0$.

For example, $C_{2}=T\left(C_{1}\right)+C_{1} x_{0}^{-1} T\left(C_{0}\right)$ and $C_{3}=T\left(C_{2}\right)+C_{1} x_{0}^{-1} T\left(C_{1}\right)+C_{2} x_{0}^{-1} T\left(C_{0}\right)$.

The following is an immediate corollary of Proposition 2.3 .

Corollary 2.4. The formal power series $\mathbf{C}(t)=\sum_{n=0}^{\infty} C_{n} t^{n} \in(\mathbb{Z} F)[[t]]$ satisfies:

$$
\mathbf{C}(t)=x_{0}+t \mathbf{C}(t) x_{0}^{-1} T(\mathbf{C}(t)), T(\mathbf{C}(t)) x_{0}^{-1} \mathbf{C}(t)=\mathbf{C}(t) x_{0}^{-1} T(\mathbf{C}(t))
$$

Remark 2.5. Applying $\varepsilon$ to (2.5), we obtain the well-known functional equation $c(t)=1+t c(t)^{2}$ for the classical generating function $c(t)=\sum_{n=0}^{\infty} \varepsilon\left(C_{n}\right) t^{n}$ of Catalan numbers.

Remark 2.6. After the first version of this paper became available, Philippe Di Francesco and Rinat Kedem pointed to us that $\mathbf{C}(t) x_{0}^{-1}$ is a noncommutative Stieltjes continued fraction which can be computed by combining methods of [8, Section 3.3.1] and [12, Section 8] as follows.

$$
\mathbf{C}(t) x_{0}^{-1}=\lim _{k \rightarrow \infty} \mathbf{S}\left(x_{1} x_{0}^{-1}, \ldots, x_{k} x_{k-1}^{-1}, t\right),
$$

where $\mathbf{S}\left(z_{1}, t\right)=\left(1-z_{1} t\right)^{-1}, \mathbf{S}\left(z_{1}, \ldots, z_{k}, t\right)=\mathbf{S}\left(z_{1}, \ldots, z_{k-2}, \mathbf{S}\left(z_{k}, t\right) z_{k-1}, t\right)$ for $k \geq 2$.

Remark 2.7. In fact, there is another recursion

$$
C_{n+1}=C_{n} x_{0}^{-1} x_{1}+\sum_{k=1}^{n} C_{k} x_{1}^{-1} T^{2}\left(C_{n-k}\right)=x_{1} x_{0}^{-1} C_{n}+\sum_{k=0}^{n-1} T^{2}\left(C_{k}\right) x_{1}^{-1} C_{n-k}
$$

for $n \geq 1$. For instance, $C_{3}=C_{2} x_{0}^{-1} x_{1}+C_{1} x_{1}^{-1} T^{2}\left(C_{1}\right)+C_{2} x_{1}^{-1} T^{2}\left(C_{0}\right)=C_{2} x_{0}^{-1} x_{1}+x_{3}+C_{2} x_{1}^{-1} x_{2}$. The recursion leads to the functional equation $\mathbf{C}(t)=x_{0}+t\left(\mathbf{C}(t) x_{0}^{-1} x_{1}-x_{0} x_{1}^{-1} T^{2}(\mathbf{C}(t))+\mathbf{C}(t) x_{1}^{-1} T^{2}(\mathbf{C}(t))\right)$, which we leave as an exercise to the reader. 
Remark 2.8. Equations (2.4) can be written in a matrix form: $H x_{0}^{-1} T(H)=T(H) x_{0}^{-1} H=H^{\prime}$, where $H$ (resp. $H^{\prime}$ ) is the lower triangular $\mathbb{Z}_{\geq 0} \times \mathbb{Z}_{\geq 0}$ Toeplitz matrix whose $\left(i, j\right.$ )-th entry is $C_{i-j}$ (resp. $C_{i-j+1}$ ) if $i \geq j$. Thus, $H^{-1}$ is a lower triangular Toeplitz matrix whose $(i, j)$-th entry is $-x_{0}^{-1} T\left(C_{i-j-1}\right) x_{0}^{-1}$ for $i>j$.

It turns out that there is a remarkable specialization $\underline{C}_{n} \in \mathbb{Z} F_{1}$ of $C_{n}$. Indeed, let $\sigma: \mathbb{Z} F \rightarrow \mathbb{Z} F_{1}$ be a ring homomorphism given by $\sigma\left(x_{k}\right)=x_{0}^{k} x_{1}^{k}, k \in \mathbb{Z}_{\geq 0}$. Abbreviate $\underline{C}_{n}:=\sigma\left(C_{n}\right)$ for $n \geq 0$.

The following result asserts, in particular, that $\underline{C}_{n}$ are noncommutative polynomials (rather than Laurent polynomials) and they satisfy yet another noncommutative generalization of the well-known classical recursion for Catalan numbers.

Proposition 2.9. The elements $\underline{C}_{n} \in \mathbb{Z}\left\langle x_{0}, x_{1}\right\rangle$ are determined by the following recursion: $\underline{C}_{0}=1$ and

$$
\underline{C}_{n+1}=\sum_{k=0}^{n} \underline{C}_{k} x_{0} \underline{C}_{n-k} x_{1}=\sum_{k=0}^{n} x_{0} \underline{C}_{k} x_{1} \underline{C}_{n-k},
$$

for $n \geq 0$. In particular, all $\underline{C}_{n}$ belong to the free semi-ring $\mathbb{Z}_{\geq 0}\left\langle x_{0}, x_{1}\right\rangle \subset \mathbb{Z}_{\geq 0} F_{1}$.

Our proof of the proposition is based on the identity $\sigma\left(T^{i} C_{n}\right)=x_{0}^{i} \sigma\left(C_{n}\right) x_{1}^{i}$ for $i, n \geq 0$ (see Lemma 3.3).

Remark 2.10. Applying $\sigma$ to the recursions from Remark 2.7 and using the same argument from the proof of Proposition 2.9], we obtain another recursion for $\underline{C}_{n}$ :

$$
\underline{C}_{n+1}=\underline{C}_{n} x_{0} x_{1}+\sum_{k=1}^{n} \underline{C}_{k} x_{1}^{-1} x_{0} \underline{C}_{n-k} x_{1}^{2}=x_{0} x_{1} C_{n}+\sum_{k=0}^{n-1} x_{0}^{2} \underline{C}_{k} x_{1} x_{0}^{-1} \underline{C}_{n-k} .
$$

Remark 2.11. One can show that the "two-variable" noncommutative Catalan numbers are invariant under the anti-involution of $\mathbb{Z} F_{1}$ interchanging $x_{0}$ and $x_{1}$.

In fact, we can explicitly compute each $\underline{C}_{n}$. Indeed, assign a monomial $\underline{M}_{P} \in F_{1}$ to each $P \in \mathcal{P}_{n}$ by:

$$
\underline{M}_{P}=x_{0}^{j_{0}} x_{1}^{j_{1}} x_{0}^{j_{2}} \cdots x_{1}^{j_{2 k}},
$$

where $\left(j_{0}, j_{1}, \ldots, j_{2 k}\right) \in \mathbb{Z}_{>0}^{2 k+1}$ is the sequence of jumps of the path $P$, i.e., the $r$-th northwest corner is $\left(j_{0}+j_{2}+\cdots+j_{2 r}, j_{1}+j_{3}+\cdots+j_{2 r+1}\right)$ and $r$-th southeast corner of $P$ is $\left(j_{0}+j_{2}+\cdots+j_{2 r}, j_{1}+j_{3}+\cdots+j_{2 r-1}\right)$ One can easily see that $\sigma\left(M_{P}\right)=\underline{M}_{P}$, so we obtain the following immediate corollary.

Corollary 2.12. $\underline{C}_{n}=\sum_{P \in \mathcal{P}_{n}} \underline{M}_{P}$ for all $n \geq 1$.

Example 2.13. $\underline{C}_{2}=x_{0}^{2} x_{1}^{2}+x_{0} x_{1} x_{0} x_{1}, \underline{C}_{3}=x_{0}^{3} x_{1}^{3}+x_{0}^{2} x_{1} x_{0} x_{1}^{2}+x_{0}^{2} x_{1}^{2} x_{0} x_{1}+x_{0} x_{1} x_{0}^{2} x_{1}^{2}+x_{0} x_{1} x_{0} x_{1} x_{0} x_{1}$,

$$
\begin{gathered}
\underline{C}_{4}=x_{0}^{4} x_{1}^{4}+x_{0}^{3} x_{1} x_{0} x_{1}^{3}+x_{0}^{2} x_{1}^{2} x_{0}^{2} x_{1}^{2}+x_{0}^{3} x_{1}^{2} x_{0} x_{1}^{2}+x_{0}^{2} x_{1} x_{0}^{2} x_{1}^{3}+x_{0}^{3} x_{1}^{3} x_{0} x_{1}+x_{0} x_{1} x_{0}^{3} x_{1}^{3}+x_{0}^{2} x_{1} x_{0} x_{1} x_{0} x_{1}^{2} \\
+x_{0} x_{1} x_{0}^{2} x_{1}^{2} x_{0} x_{1}+x_{0}^{2} x_{1} x_{0} x_{1}^{2} x_{0} x_{1}+x_{0} x_{1} x_{0}^{2} x_{1} x_{0} x_{1}^{2}+x_{0}^{2} x_{1}^{2} x_{0} x_{1} x_{0} x_{1}+x_{0} x_{1} x_{0} x_{1} x_{0}^{2} x_{1}^{2}+x_{0} x_{1} x_{0} x_{1} x_{0} x_{1} x_{0} x_{1} .
\end{gathered}
$$

The following immediate result is a "two-variable" version of Corollary 2.4 ,

Corollary 2.14. The formal power series $\underline{\mathbf{C}}(t)=\sum_{n=0}^{\infty} \underline{C}_{n} t^{n} \in \mathbb{Z}\left\langle x_{0}, x_{1}\right\rangle[[t]]$ satisfies:

$$
\underline{\mathbf{C}}(t)=1+t \underline{\mathbf{C}}(t) x_{0} \underline{\mathbf{C}}(t) x_{1} .
$$

Remark 2.15. For $t=1$, the equation (2.7) coincides with the quadratic equation on formal series $K\left(x_{0}, x_{1}\right)$ studied in [18] where a solution of this equation was presented as a "noncommutative Rogers-Ramanujan continued fraction".

Remark 2.16. In our previous work [5] on the inversion of $\sum_{n \geq 0} x_{0}^{n} x_{1}^{n}$ in the ring of formal series $\mathbb{Z}\left\langle\left\langle x_{0}, x_{1}\right\rangle\right\rangle$ in noncommutative variables $x_{0}, x_{1}$ we encountered a quadratic equation $D=1-D x_{0} x_{1}+D x_{0} D x_{1}$ for some $D \in \mathbb{Z}\left\langle\left\langle x_{0}, x_{1}\right\rangle\right\rangle$ and noticed that it is very similar to (2.7). This was the starting point of the project.

Remark 2.17. In fact, there is another group homomorphism $\pi: F \rightarrow F_{1}$ given by $\pi\left(x_{k}\right)=x_{0} \cdot\left(x_{0}^{-1} x_{1}\right)^{k}$, $k \in \mathbb{Z}_{\geq 0}$, which results in an "almost commutative" specialization of noncommutative Catalan numbers: $\pi\left(C_{n}\right)=\pi\left(x_{n}\right) \cdot \frac{1}{n+1}\left(\begin{array}{c}2 n \\ n\end{array}\right)$. 
For each $0 \leq k \leq n$ denote by $\mathcal{P}_{n}^{k}$, the set of all $P \in \mathcal{P}_{n}$ such that the rightmost southeast corner $p$ of $P$ satisfies $p=(n, y)$, where $y \leq k$. In particular, $\mathcal{P}_{n}^{n-1}=\mathcal{P}_{n}^{n}=\mathcal{P}_{n}$. For each $0 \leq k \leq n$ define truncated noncommutative Catalan number $C_{n}^{k} \in \mathbb{Z} F_{n}$ by

$$
C_{n}^{k}:=\sum_{P \in \mathcal{P}_{n}^{k}} M_{P}
$$

The following recursion on $C_{n}^{k}$ is immediate.

Lemma 2.18. $C_{n}^{k}=C_{n}^{k-1}+C_{n-1}^{k} x_{n-k-1}^{-1} x_{n-k}$ for all $1 \leq k \leq n$ (with the convention $C_{n}^{\ell}=0$ if $\ell>n$ ).

Example 2.19. $C_{n}^{0}=x_{n}, C_{n}^{n-1}=C_{n}^{n}=C_{n}$ for all $n \geq 1$. Also, $C_{n}^{1}=x_{n}+\sum_{i=1}^{n-1} x_{i} x_{i-1}^{-1} x_{n-1}$,

$$
C_{n}^{2}=\sum_{1 \leq i \leq j \leq n, j>1} x_{i} x_{i-1}^{-1} x_{j-1} x_{j-2}^{-1} x_{n-2} .
$$

Sometimes it is convenient to express $C_{n}^{k}$ via $y_{i}:=x_{i} x_{i-1}^{-1}, i \in \mathbb{Z}_{\geq 1}$. Indeed, denote $\tilde{C}_{n}^{k}:=C_{n}^{k} x_{n-k}^{-1}$ for $k, n \in \mathbb{Z}_{\geq 0}, k \leq n$.

The following result generalizes a number of basic properties of truncated Catalan numbers.

Proposition 2.20. For all $0 \leq k \leq n$ one has:

(a) $\tilde{C}_{n}^{k}=\sum_{j_{1} \leq \ldots \leq j_{k} \leq n: j_{1} \geq 1, \ldots, j_{k} \geq k} y_{j_{1}} y_{j_{2}-1} \ldots y_{j_{k}-k+1}$.

(b) $\tilde{C}_{n}^{k}=\tilde{C}_{n-1}^{k}+\tilde{C}_{n}^{k-1} y_{n+1-k}$ (with the convention $\tilde{C}_{n}^{\ell}=0$ if $\ell>n$ ).

(c) $\tilde{C}_{n+1}^{k}=\sum_{i=0}^{k} \tilde{C}_{i}^{i} T\left(\tilde{C}_{n-i}^{k-i}\right)$.

A proof follows from Lemmas 3.1, 3.2.

Example 2.21. $\tilde{C}_{n}^{0}=1, \tilde{C}_{n}^{1}=y_{1}+\cdots+y_{n}$, and $\tilde{C}_{n}^{n}=\tilde{C}_{n}^{n-1} y_{1}$ for all $n \geq 1$.

$$
\tilde{C}_{n}^{2}=\sum_{1 \leq i \leq j \leq n, j>1} y_{i} y_{j-1}, \tilde{C}_{n}^{3}=\sum_{1 \leq i \leq j \leq k \leq n, j>1, k>2} y_{i} y_{j-1} y_{k-2} .
$$

However, the following recursion is rather non-trivial (and we could not find its classical analogue in the literature).

Theorem 2.22. $C_{n}=\sum_{\substack{a, b \in \mathbb{Z} \\ a+b \leq n, a-b=d}} C_{n-b}^{a} x_{n-a-b}^{-1} \overline{C_{n-a}^{b}}$ for each $n \in \mathbb{Z}_{\geq 0}$ and each $d \in \mathbb{Z}$ with $|d| \leq n$ (e.g., the right hand side does not depend on $d)$.

A proof is given by Lemmas 3.43 .6 in Section 3.1

Remark 2.23. In particular, Theorem 2.22 provides another confirmation "-invariance of noncommutative Catalan numbers (established in Proposition 2.1).

It turns out that the above "two-variable specialization" $\sigma$ is also of interest for truncated noncommutative Catalan numbers. Indeed, in the notation as above, denote $\underline{C}_{n}^{k}:=\sigma\left(C_{n}^{k}\right)$ and $\underline{\underline{C}}_{n}^{k}:=\underline{C}_{n}^{k} x_{1}^{k-n}$.

The following is immediate.

Corollary 2.24. In the notation of Proposition [2.9, one has

(a) $\underline{C}_{n}^{k}=\sum_{P \in \mathcal{P}_{n}^{k}} \underline{M}_{P}$ for all $k, n \in \mathbb{Z}_{\geq 0}, k \leq n$.

(b) $\underline{\underline{C}}_{n}^{k}=\underline{\underline{C}}_{n}^{k-1} x_{1}+\underline{\underline{C}}_{n-1}^{k} x_{0}$ for all $1 \leq k \leq n$ (with the convention $\underline{\underline{C}}_{n}^{\ell}=0$ if $\ell>n$ ). In particular, each $\underline{\underline{C}}_{n}^{k}$ is a noncommutative polynomial in $x_{0}, x_{1}$ of degree $n+k$.

Example 2.25. $\underline{\underline{C}}_{n}^{0}=x_{0}^{n}, \underline{\underline{C}}_{n}^{1}=x_{0}^{n} x_{1}+\sum_{i=1}^{n-1} x_{0}^{i} x_{1} x_{0}^{n-i}, \underline{\underline{C}}_{n}^{2}=\underline{\underline{C}}_{n}^{1} x_{1}+\sum_{1 \leq i \leq j \leq n-1, j>1} x_{0}^{i} x_{1} x_{0}^{j-i} x_{1} x_{0}^{n-j}$. 
It turns out that our (truncated) noncommutative Catalan numbers $\tilde{C}_{n}^{k}$ admit another specialization into certain polynomials in $\mathbb{Z}_{\geq 0}[q]$ defined by Garsia and Haiman in [10. Namely, let $\chi_{q}: \mathbb{Z} F \rightarrow \mathbb{Z}\left[q, q^{-1}\right]$ be a ring homomorphism defined by $\chi_{q}\left(x_{k}\right)=q^{\frac{k(k-1)}{2}}$ for $k \geq 0$, i.e., $\chi_{q}\left(y_{k}\right)=q^{k-1}$ for $k \in \mathbb{Z}_{\geq 1}$.

Define polynomials $c_{n}^{k}(q, t) \in \mathbb{Z}_{\geq 0}[q, t], 0 \leq k \leq n$ recursively by $c_{n}^{0}(q, t)=1$ and

$$
c_{n}^{k}(q, t)=\sum_{r=1}^{k}\left[\begin{array}{c}
r+n-k \\
r
\end{array}\right]_{q} t^{k-r} q^{\frac{r(r-1)}{2}} c_{k-1}^{k-r}(q, t)
$$

where $\left[\begin{array}{l}n \\ k\end{array}\right]_{q}$ denotes the $q$-binomial coefficient $\frac{[n]_{q} !}{\left.[k]_{q} ! n-k\right]_{q} !},[n]_{q} !=[1]_{q} \cdots[n]_{q},[k]_{q}=\frac{1-q^{n}}{1-q}=1+q+\cdots q^{k-1}$.

These polynomials are closely related to polynomials $H_{n, k}(q, t)$ introduced by Garsia and Haglund ([11, Equation I.24]), namely, $c_{n}^{k}(q, t)=t^{-k} q^{-\frac{(n+1-k)(n-k)}{2}} H_{n+1, n+1-k}(q, t)$, in particular, $c_{n}^{n}(q, t)=c_{n}(q, t)$ is the celebrated $(q, t)$-Catalan number introduced in [10].

The following result shows that our (truncated) noncommutative Catalan numbers are noncommutative deformations of $(q, 1)$-Catalan numbers.

Theorem 2.26. $\chi_{q}\left(\tilde{C}_{n}^{k}\right)=c_{n}^{k}(q, 1)$ for all $k \leq n$, in particular, $\chi_{q}\left(C_{n}\right)=c_{n}(q, 1)$ for $n \geq 0$.

We prove Theorem 2.26 in Section 3.1 .

Example 2.27. $\chi_{q}\left(\tilde{C}_{n}^{1}\right)=[n+1]_{q}$ and $\chi_{q}\left(\tilde{C}_{n}^{k}\right)=\chi_{q}\left(\tilde{C}_{n}^{k-1}\right) q^{n-k}+\chi_{q}\left(\tilde{C}_{n-1}^{k}\right)$ for $1 \leq k \leq n$.

Remark 2.28. It is curious that for another class of $q$-Catalan numbers, $q^{\frac{n(n-1)}{2}} c_{n}\left(q, q^{-1}\right)=\frac{1}{[n+1]_{q}}\left[\begin{array}{c}2 n \\ n\end{array}\right]_{q}$, there is no analogue of Theorem 2.26. Also, it would be interesting to find an appropriate noncommutative deformations of $(q, t)$-Catalan numbers.

The following result is a generalization of the well-known property of Hankel determinants of $q$-Catalan numbers.

Theorem 2.29. For $n \geq 1, m \in\{0,1\}$ the determinant of the $(n+1) \times(n+1)$ matrix $\left(c_{i+j+m}(q, 1)\right)$, $i, j=0, \ldots, n$, is $q^{\frac{n(n+1)(4 n-1+6 m)}{6}}$.

We prove Theorem 2.29 in Section 3.4 .

Define the noncommutative binomial coefficients $\left(\begin{array}{l}n \\ k\end{array}\right) \in \mathbb{Z} F_{n+k-1},\left(\begin{array}{l}n \\ k\end{array}\right)^{\prime} \in \mathbb{Z} F_{n}$ by

$$
\left(\begin{array}{l}
n \\
k
\end{array}\right)=\sum y_{J},\left(\begin{array}{l}
n \\
k
\end{array}\right)^{\prime}=\sum y_{J}^{\prime}
$$

where each summation is over all subsets $J=\left\{j_{1}<j_{2}<\cdots<j_{k}\right\}$ of $[1, n]$ and we abbreviated $y_{J}=$ $y_{j_{k}+k-1} \cdots y_{j_{2}+1} y_{j_{1}}, y_{J}^{\prime}=y_{j_{1}+k-1} y_{j_{2}+k-3} \cdots y_{j_{k}+1-k}$ for $j \in \mathbb{Z}_{\geq 1}$.

Remark 2.30. The $q$-binomial coefficients can be expressed as $\left[\begin{array}{l}n \\ k\end{array}\right]_{q}=\sum q^{j_{1}+\cdots+j_{k}-\frac{k(k+1)}{2}}$, where the summation is over all subsets $J=\left\{j_{1}<j_{2}<\cdots<j_{k}\right\}$ of $[1, n]$. Therefore, under the above specialization $\chi_{q}: \mathbb{Z} F \rightarrow \mathbb{Z}\left[q, q^{-1}\right]$ we have $\left.\chi_{q}\left(\left(\begin{array}{l}n \\ k\end{array}\right)\right)=q^{k(k-1)}\left[\begin{array}{l}n \\ k\end{array}\right]_{q}, \chi_{q}\left(\left(\begin{array}{l}n \\ k\end{array}\right)\right)=q^{\prime}\right)^{\frac{k(k-1)}{2}}\left[\begin{array}{l}n \\ k\end{array}\right]_{q}$ for all $k, n \in \mathbb{Z}_{\geq 0}$.

Example 2.31. $\left(\begin{array}{l}n \\ 0\end{array}\right)=\left(\begin{array}{l}n \\ 0\end{array}\right)^{\prime}=1,\left(\begin{array}{c}n \\ 1\end{array}\right)=\left(\begin{array}{l}n \\ 1\end{array}\right)^{\prime}=\sum_{i=1}^{n} y_{i},\left(\begin{array}{l}n \\ 2\end{array}\right)=\sum_{1 \leq i<j \leq n} y_{j+1} y_{i},\left(\begin{array}{l}n \\ n\end{array}\right)=y_{2 n-1} \cdots y_{3} y_{1}=y_{[1, n]}$, $\left(\begin{array}{c}n \\ n-1\end{array}\right)=\sum_{i=1}^{n} y_{[1, n] \backslash\{i\}},\left(\begin{array}{c}n \\ n-2\end{array}\right)=\sum_{1 \leq i<j \leq n} y_{[1, n] \backslash\{i, j\}},\left(\begin{array}{l}n \\ 2\end{array}\right)^{\prime}=\sum_{1 \leq i<j \leq n} y_{i+1} y_{j-1},\left(\begin{array}{l}n \\ n\end{array}\right)^{\prime}=y_{n} y_{n-1} \cdots y_{1}=y_{[1, n]}^{\prime}$, $\left(\begin{array}{c}n \\ n-1\end{array}\right)^{\prime}=\sum_{i=1}^{n} y_{[1, n] \backslash\{i\}}^{\prime},\left(\begin{array}{c}n \\ n-2\end{array}\right)^{\prime}=\sum_{1 \leq i<j \leq n} y_{[1, n] \backslash\{i, j\}}^{\prime}$. 
Clearly, $\varepsilon\left(\left(\begin{array}{l}n \\ k\end{array}\right)\right)=\varepsilon\left(\left(\begin{array}{l}n \\ k\end{array}\right)^{\prime}\right)=\left(\begin{array}{l}n \\ k\end{array}\right)$ and $\left(\begin{array}{l}n \\ k\end{array}\right)=\left(\begin{array}{l}n \\ k\end{array}\right)^{\prime}=0$ if $k \notin[0, n]$.

Similarly to the classical case, we have an analogue of the Pascal triangle and the multiplication law for noncommutative binomial coefficients.

Theorem 2.32. $\left(\begin{array}{c}m+n \\ k\end{array}\right)=\sum_{\substack{a, b \in \mathbb{Z}_{\geq 0}: \\ a+b=k}} T^{n+b}\left(\left(\begin{array}{c}m \\ a\end{array}\right)\right)\left(\begin{array}{l}n \\ b\end{array}\right),\left(\begin{array}{c}m+n \\ k\end{array}\right)^{\prime}=\sum_{\substack{a, b \in \mathbb{Z}_{\geq 0}: \\ a+b=k}} T^{b}\left(\left(\begin{array}{c}m \\ a\end{array}\right)\right)^{\prime} T^{m-a}\left(\left(\begin{array}{c}n \\ b\end{array}\right)^{\prime}\right)$ for $m, n, k$ $\in \mathbb{Z}_{\geq 0}$. In particular, $\left(\begin{array}{c}n+1 \\ k\end{array}\right)=\left(\begin{array}{l}n \\ k\end{array}\right)+y_{n+k}\left(\begin{array}{c}n \\ k-1\end{array}\right),\left(\begin{array}{c}n+1 \\ k\end{array}\right)^{\prime}=T\left(\left(\begin{array}{l}n \\ k\end{array}\right)^{\prime}\right)+y_{k}\left(\left(\begin{array}{c}n \\ k-1\end{array}\right)^{\prime}\right)$ for all $n, k \in \mathbb{Z}_{\geq 0}$.

Actually, Theorem 2.32 which is proved in Section 3.2 together with the recursion from Proposition 2.20(b) imply the following analogue of the multiplication law for the truncated noncommutative Catalan numbers, which justified the introduction of noncommutative binomial coefficients of the "second kind."

Corollary 2.33. $\tilde{C}_{m+n}^{k}=\sum_{\ell=0}^{n} \tilde{C}_{m+\ell}^{k-\ell} \cdot T^{m-k+\ell}\left(\left(\begin{array}{l}n \\ \ell\end{array}\right)^{\prime}\right)$ for all $m, n, k \in \mathbb{Z}_{\geq 0}$.

The following relation between truncated noncommutative Catalan numbers and the binomial coefficients of the "first kind" is rather surprising.

Theorem 2.34. $\sum_{j=0}^{k}(-1)^{j} \tilde{C}_{n+k-j}^{j} \cdot\left(\begin{array}{l}n-j \\ k-j\end{array}\right)=0$ for any $0<k \leq n$.

We prove Theorem 2.34 in Section 3.2 (Lemmas 3.7 3.8).

Remark 2.35. In fact, there is an accompanying identity $\sum_{j=0}^{k}(-1)^{j}\left(\begin{array}{c}n+k-j \\ j\end{array}\right) \cdot \tilde{C}_{n-j}^{k-j}=0$ for any $0<k \leq n$, which follows from Theorem 2.43 below. We leave this as an exercise to the readers.

This turns out to be equivalent to the following "determinantal" identities between noncommutative truncated Catalan numbers and binomial coefficients (whose classical analogues also seem to be new).

Theorem 2.36. For all $k, n \in \mathbb{Z}_{\geq 0}, k \leq n$ one has $\tilde{C}_{n}^{k}=\sum_{J}(-1)^{k+1-|J|} M_{n, J},\left(\begin{array}{l}n \\ k\end{array}\right)=\sum_{J}(-1)^{k+1-|J|} \tilde{M}_{n, J}$, where each summation is over all subsets $J=\left\{0=j_{0}<\cdots<j_{\ell}=k\right\}$ of $[0, k]$ and

$$
\begin{gathered}
M_{n, J}=\left(\begin{array}{c}
n+j_{\ell-1}+j_{\ell}-k \\
j_{\ell}-j_{\ell-1}
\end{array}\right) \cdots\left(\begin{array}{c}
n+j_{1}+j_{2}-k \\
j_{2}-j_{1}
\end{array}\right)\left(\begin{array}{c}
n+j_{0}+j_{1}-k \\
j_{1}-j_{0}
\end{array}\right), \\
\tilde{M}_{n, J}=\tilde{C}_{n+j_{0}+j_{1}-k}^{j_{1}-j_{0}} \cdot \tilde{C}_{n+j_{1}+j_{2}-k}^{j_{2}-j_{1}} \cdots \tilde{C}_{n+j_{\ell-1}+j_{\ell}-k}^{j_{\ell-j_{\ell-1}}} .
\end{gathered}
$$

We prove Theorem 2.36 in Section 3.4 .

Actually, Theorems 2.26, 2.34, and 2.36 hint to some remarkable properties of Hankel matrices with noncommutative Catalan numbers as entries.

For $m \in \mathbb{Z}_{\geq 0}$ define the $\mathbb{Z}_{\geq 0} \times \mathbb{Z}_{\geq 0}$ matrix $H_{m}$ over $\mathbb{Z} F$ whose $(i, j)$-th entry is $C_{m+i+j}, i, j \in \mathbb{Z}_{\geq 0}$ and for each $n \geq 0$ denote by $H_{m, n}$ the principal $[0, n] \times[0, n]$ submatrix of $H_{m}$.

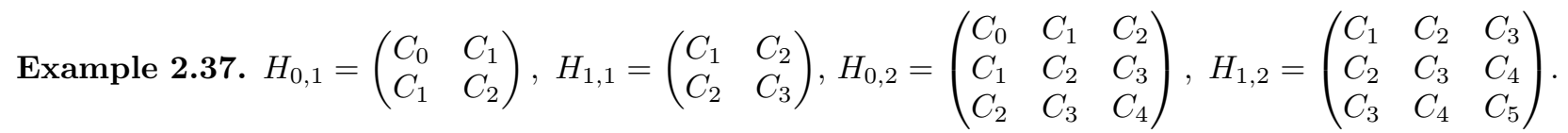

We refer to all $H_{m}$ and $H_{m}^{n}$ as noncommutative Hankel-Catalan matrices by analogy with its classical counterpart $\varepsilon\left(H_{m, n}\right) \in \operatorname{Mat}_{n+1, n+1}(\mathbb{Z})$.

We will finish the section by showing that each $H_{m, n}, m \in\{0,1\}, n \geq 0$ admits a Gauss factorization over $\mathbb{Z} F$ involving truncated noncommutative Catalan numbers and it inverse (which is also a matrix over $\mathbb{Z} F$ ) is given by an interesting combinatorial formula involving our noncommutative binomial coefficients.

For $m \in\{0,1\}$ let $L_{m}$ be the lower unitriangular $\mathbb{Z}_{\geq 0} \times \mathbb{Z}_{\geq 0}$ matrix whose $(j, i)$-th entry, $0 \leq i \leq j$, is $\tilde{C}_{i+j+m}^{j-i}$ and let $U_{m}$ be the upper triangular $\mathbb{Z}_{\geq 0} \times \mathbb{Z}_{\geq 0}$ matrix whose $(i, j)$-th entry, $0 \leq i \leq j$, is $\overline{C_{i+j+m}^{j-i}}$ ). 
Theorem 2.38. $H_{m}=L_{m} \cdot U_{m}$ for each $m \in\{0,1\}$.

We prove Theorem 2.38 in Section 3.3 .

Remark 2.39. A classical version of this result, $\varepsilon\left(H_{m}\right)=\varepsilon\left(L_{m}\right) \cdot \varepsilon\left(U_{m}\right)$, was established in [1].

Theorem 2.38 and [13, Theorem 4.9.7] imply the following immediate corollary.

Corollary 2.40. $C_{m+i+j}^{j-i}$ equals the quasidetermiant $\left|\begin{array}{cccc}C_{m} & C_{m+1} & \ldots & C_{m+i} \\ C_{m+1} & C_{m+2} & \ldots & C_{m+i+1} \\ & & \ldots & \\ C_{m+i-1} & C_{m+i} & \ldots & C_{m+2 i-1} \\ C_{m+j} & C_{m+j+1} & \ldots & C_{m+i+j}\end{array}\right|$ for $0 \leq i \leq j$, $m \in\{0,1\}$ (see [14, 15] for notation). In particular,

$$
\left|\begin{array}{cccc}
C_{m} & C_{m+1} & \cdots & C_{m+n} \\
C_{m+1} & C_{m+2} & \cdots & C_{m+n+1} \\
& & \cdots & \\
C_{m+n} & C_{m+n+1} & \cdots & C_{m+2 n}
\end{array}\right|=x_{m+2 n}
$$

for all $n \in \mathbb{Z}_{\geq 0}, m \in\{0,1\}$.

Remark 2.41. In fact, (2.8) is noncommutative generalization of the well-known fact that $\operatorname{det}\left(\varepsilon\left(H_{0, n}\right)\right)=$ $\operatorname{det}\left(\varepsilon\left(H_{1, n}\right)\right)=1$ for $n \geq 0$. Moreover similarly to the classical case, noncommutative Catalan numbers are uniquely determined by equations (2.8) for $n \in \mathbb{Z}_{\geq 0}, m \in\{0,1\}$.

Remark 2.42. Noncommutative Hankel quasideterminants were introduced in $[12]$ in the context of inversion of noncommutative power series. In fact, [12, Corollary 8.3] asserts that such an inverse can be expressed via continued fractions involving such quasideterminants of the coefficients of the series in question. This correlates with Remark 2.6 above.

For $m \in\{0,1\}$ let $L_{m}^{-}$be the lower unitriangular $\mathbb{Z}_{\geq 0} \times \mathbb{Z}_{\geq 0}$ matrix whose $(j, i)$-th entry, $0 \leq i \leq j$, is $(-1)^{i+j}\left(\begin{array}{c}i+j+m \\ j-i\end{array}\right)$ and let $U_{m}^{-}$be the upper triangular $\mathbb{Z}_{\geq 0} \times \mathbb{Z}_{\geq 0}$ matrix whose $(i, j)$-th entry, $0 \leq i \leq j$, is $(-1)^{i+j} \overline{\left(\begin{array}{c}i+j+m \\ j-i\end{array}\right)} x_{2 j+m}^{-1}$.

For any $\mathbb{Z}_{\geq 0} \times \mathbb{Z}_{\geq 0}$ matrix $M$ denote by $\left.M\right|_{n}$ the principal $(n+1) \times(n+1)$-submatrix of $M$ (e.g., $\left.H_{m, n}=\left.H_{m}\right|_{n}\right)$.

Theorem 2.43. $\left(U_{m}\right)^{-1}=U_{m}^{-}$and $\left(L_{m}\right)^{-1}=L_{m}^{-}$, hence $\left(H_{m, n}\right)^{-1}=\left.\left.U_{m}^{-}\right|_{n} \cdot L_{m}^{-}\right|_{n}$ for $m \in\{0,1\}, n \geq 1$.

Remark 2.44. Similar to Remark 2.39 the classical version of this result, $\varepsilon\left(H_{m, n}\right)^{-1}=\left.\varepsilon\left(\left.L_{m}^{-}\right|_{n}\right) \cdot \varepsilon\left(U_{m}^{-}\right)\right|_{n}$, seems to be new.

Computation of $H_{m}^{-1}$ for $m \geq 2$ is a more challenging task, which we will perform elsewhere.

\section{Proofs of MAIN RESUlts}

3.1. Proof of Propositions 2.3, 2.9, 2.20 and Theorems 2.22, 2.26. We start with a proof of Proposition 2.20, Then specializations will lead to Propositions 2.3 and 2.9.

Proof of Proposition 2.20 Prove (a) first. Denote by $\mathbf{J}_{n}^{k}$ the set of all sequences $\mathbf{j}=\left(j_{1}, \ldots, j_{k}\right) \in \mathbb{Z}^{k}$ such that $j_{1} \leq \ldots \leq j_{k} \leq n$ and $j_{1} \geq 1, \ldots, j_{k} \geq k$.

For each $P \in \mathcal{P}_{n}^{k}$ and $s \in[1, k]$ denote by $j_{s}(P)$ the minimum of $x$-coordinates of all points in $P$ whose $y$-coordinate is $s$. For each $\mathbf{j}=\left(j_{1}, \ldots, j_{k}\right) \in \mathbb{Z}^{k}$ with $j_{s} \geq s, s \in[1, k]$ we abbreviate $y_{\mathbf{j}}=y_{j_{1}} y_{j_{2}-1} \ldots y_{j_{k}-k+1}$.

The following is immediate.

Lemma 3.1. For all $k, n \in \mathbb{Z}_{\geq 0}, k \leq n$ one has:

(a) The assignments $P \mapsto \mathbf{j}(P):=\left(j_{1}(P), \ldots, j_{k}(P)\right)$ defines a bijection $\mathcal{P}_{n}^{k} \widetilde{\neg} \mathbf{J}_{n}^{k}$.

(b) For each $P \in \mathcal{P}_{n}^{k}$ we have $M_{P} x_{n-k}^{-1}=y_{\mathbf{j}(P)}$. 
Using Lemma 3.1(b), we obtain $\tilde{C}_{n}^{k}=\sum_{\mathbf{j} \in \mathbf{J}_{n}^{k}} y_{\mathbf{j}}$ and thus finish the proof of (a).

Prove (b). It is easy to see that $\mathbf{J}_{n}^{k}=\mathbf{J}_{n-1}^{k} \sqcup\left(\mathbf{J}_{n}^{k-1}, n\right)$. Therefore,

$$
\tilde{C}_{n}^{k}=\sum_{\mathbf{j} \in \mathbf{J}_{n}^{k}} y_{\mathbf{j}}=\sum_{\mathbf{j} \in \mathbf{J}_{n}^{k}} y_{\mathbf{j}}+\sum_{\mathbf{j} \in\left(\mathbf{J}_{n}^{k-1}, n\right)} y_{\mathbf{j}}=\tilde{C}_{n-1}^{k}+\tilde{C}_{n}^{k-1} y_{n+1-k} .
$$

This proves (b).

To prove (c) we need the following result.

Lemma 3.2. $\mathbf{J}_{n+1}^{k}=\bigsqcup_{i=0}^{k} \mathbf{J}_{i}^{i} \times T^{i+1}\left(\mathbf{J}_{n-i}^{k-i}\right)$ for all $k, n \in \mathbb{Z}_{\geq 0}, 0 \leq k \leq n$, where $T=T_{r}: \mathbb{Z}^{r} \rightarrow \mathbb{Z}^{r}, r \geq 1$ is the translation given by $x \mapsto x+\underbrace{(1, \ldots, 1)}_{r}$.

Proof. For each $\mathbf{j}=\left(j_{1}, \ldots, j_{k}\right) \in \mathbf{J}_{n+1}^{k}$ denote by $i_{\mathbf{j}}$ the largest $i \in[1, k]$ such that $j_{i}=i$ and set $i(\mathbf{j}):=0$ if such an $i$ does not exist. This implies that, $\left\{\mathbf{j} \in \mathbf{J}_{n+1}^{k}: i_{\mathbf{j}}=i\right\}=\mathbf{J}_{i}^{i} \times T^{i+1}\left(\mathbf{J}_{n-i-1}^{k-i}\right)$ for all $i \in[0, k]$ (the first factor is empty for $i=0$ ).

The lemma is proved.

Taking into account that for $\mathbf{j}=\left(\mathbf{j}^{\prime}, T^{i+1}\left(\mathbf{j}^{\prime \prime}\right)\right) \in \mathbf{J}_{i}^{i} \times T^{i+1}\left(\mathbf{J}_{n-i}^{k-i}\right)$, we have $y_{\mathbf{j}}=y_{\mathbf{j}^{\prime}} T\left(y_{\mathbf{j}^{\prime \prime}}\right)$, we obtain:

$$
\tilde{C}_{n+1}^{k}=\sum_{\mathbf{j} \in \mathbf{J}_{n+1}^{k}} y_{\mathbf{j}}=\sum_{i \in[0, k], \mathbf{j}^{\prime} \in \mathbf{J}_{i}^{i}, \mathbf{j}^{\prime \prime} \in \mathbf{J}_{n-i}^{k-i}} y_{\mathbf{j}^{\prime}} T\left(y_{\mathbf{j}^{\prime \prime}}\right)=\sum_{i=0}^{k} \tilde{C}_{i}^{i} T\left(\tilde{C}_{n-i}^{k-i}\right) .
$$

This proves (c).

Proposition 2.20 is proved.

Proof of Theorem 2.26 Applying $\chi_{q}$ to $\tilde{C}_{n+1}^{k}$ given by Proposition 2.20(c) and using the fact that $\chi_{q}(T(y))=q^{d} \chi_{q}(y)$ for any homogeneous noncommutative polynomial of degree $d$ in $y_{1}, y_{2}, \ldots$, we obtain:

$$
\chi_{q}\left(\tilde{C}_{n+1}^{k}\right)=\sum_{i=0}^{k} q^{k-i} \chi_{q}\left(\tilde{C}_{i}^{i}\right) \chi_{q}\left(\tilde{C}_{n-i}^{k-i}\right)
$$

for all $0 \leq k \leq n$. In view of [16, Equation (3.41)] and that $F_{n, k}(q, t)=H_{n, k}(q, t)=t^{n-k} q^{\frac{k(k-1)}{2}} c_{n-1}^{n-k}(q, t)$ for all $0 \leq k<n$, we obtain same recursion $c_{n+1}^{k}=\sum_{i=0}^{k} c_{i}^{i}(q, 1) q^{k-i} c_{n-i}^{k-i}(q, 1)$ for all $0 \leq k \leq n$. Using this and taking into account that $\chi_{q}\left(\tilde{C}_{n+1}^{n}\right)=\chi_{q}\left(\tilde{C}_{n+1}^{n+1}\right)$, we conclude that $\chi_{q}\left(\tilde{C}_{n}^{k}\right)=c_{n}^{k}(q, 1)$ for all $0 \leq k \leq n$.

The theorem is proved.

Proof of Proposition 2.3. Indeed, taking into account that $C_{r}=\tilde{C}_{r}^{r} \cdot x_{0}=\tilde{C}_{r}^{r-1} y_{1} x_{0}$ for all $r \geq 1$, we see that the first identity (2.4) is equivalent to $\tilde{C}_{n+1}^{n}=\sum_{k=0}^{n} \tilde{C}_{k}^{k} T\left(\tilde{C}_{n-k}^{n-k}\right)$ which coincides with the assertion of Proposition 2.20(c) with $k=n$.

The second identity (2.4) follows from the first one and Proposition 2.1 by applying the anti-involution - .

Proposition 2.3 is proved.

Proof of Proposition 2.9. We say that $x \in F$ is alternating if it is of the form $x_{i_{1}} x_{i_{2}}^{-1} x_{i_{3}} \ldots x_{i_{s-1}}^{-1} x_{i_{s}}$ for some $i_{1}, \ldots, i_{s} \in \mathbb{Z}_{>0}$ and denote by $F^{\text {alt }}$ the set of all alternating elements in $F$. We also denote by $\mathbb{Z} F^{a l t}$ the $\mathbb{Z}$-linear span of $F^{\text {alt }}$ in $\mathbb{Z} F$. We need the following fact.

Lemma 3.3. $\sigma(T(x))=x_{0} \sigma(x) x_{1}$ for all $x \in \mathbb{Z} F^{\text {alt }}$.

Proof. We first prove the assertion for all $x \in F^{a l t}$. Indeed,let $x=x_{i_{1}} x_{i_{2}}^{-1} x_{i_{3}} \ldots x_{i_{s-1}}^{-1} x_{i_{s}}$ for some $i_{1}, i_{2}, \ldots, i_{s} \geq 0$. We have $\sigma(T(x))=\sigma\left(x_{i_{1}+1} x_{i_{2}+1}^{-1} x_{i_{3}+1} \ldots x_{i_{s-1}+1}^{-1} x_{i_{s}+1}\right)$

$$
\begin{aligned}
& =\left(x_{0}^{i_{1}+1} x_{1}^{i_{1}+1}\right)\left(x_{0}^{i_{2}+1} x_{1}^{i_{2}+1}\right)^{-1}\left(x_{0}^{i_{3}+1} x_{1}^{i_{3}+1}\right) \ldots\left(x_{0}^{i_{s-1}+1} x_{1}^{i_{s-1}+1}\right)^{-1}\left(x_{0}^{i_{s}+1} x_{1}^{i_{s}+1}\right) \\
& =x_{0} \cdot\left(x_{0}^{i_{1}} x_{1}^{i_{1}}\right)\left(x_{0}^{i_{2}} x_{1}^{i_{2}}\right)^{-1}\left(x_{0}^{i_{3}} x_{1}^{i_{3}}\right) \ldots\left(x_{0}^{i_{s-1}} x_{1}^{i_{s-1}}\right)^{-1}\left(x_{0}^{i_{s}} x_{1}^{i_{s}}\right) \cdot x_{1}=x_{0} \sigma(x) x_{1} .
\end{aligned}
$$


By linearity of $\sigma$ we obtain the assertion for all $x \in \mathbb{Z} F^{\text {alt }}$.

The lemma is proved.

Since each $C_{k}$ belongs to $\mathbb{Z} F^{\text {alt }}$, Lemma 3.3 implies that $\sigma\left(T\left(C_{k}\right)\right)=x_{0} \sigma\left(C_{k}\right) x_{1}=x_{0} \underline{C}_{k} x_{1}$ for all $k \geq 0$. Using this and applying $\sigma$ to the first identity (2.4), we obtain (2.6).

Proposition 2.9 is proved.

Proof of Theorem 2.22, In the notation of the proof of Proposition 2.20, for all $0 \leq k \leq n$ denote by $\overline{\mathbf{J}_{n}^{k}}$ the set of all $\mathbf{j}=\left(j_{1}, \ldots, j_{n}\right) \in \mathbf{J}_{n}^{n}$ such that $j_{1} \geq n-k$.

Lemma 3.4. $\bar{C}_{n}^{k} \cdot x_{0}^{-1}=\sum_{\mathbf{j} \in \overline{\mathbf{J}_{n}^{k}}} y_{\mathbf{j}}$ for all $0 \leq k \leq n$.

Proof. Indeed, in view of (2.3), we obtain using Lemma 3.1(b):

$$
\overline{C_{n}^{k}} x_{0}^{-1}=\sum_{P \in \mathcal{P}_{n}^{k}} \bar{M}_{P} \cdot x_{0}^{-1}=\sum_{P \in \mathcal{P}_{n}^{k}} M_{s_{n}(P)} \cdot x_{0}^{-1}=\sum_{P \in \mathcal{P}_{n}^{k}} y_{\mathbf{j}\left(s_{n}(P)\right)}=\sum_{\mathbf{j} \in \overline{\mathbf{J}}_{n}^{k}} y_{\mathbf{j}}
$$

because $\overline{\mathbf{J}_{n}^{k}}=\mathbf{j}\left(s_{n}\left(\mathcal{P}_{n}^{k}\right)\right)$.

The lemma is proved.

Furthermore, after multiplying by $x_{0}^{-1}$ on the right, the assertion of Theorem 2.22 is equivalent to:

$$
\tilde{C}_{n}^{n}=\sum_{\substack{a, b \in \mathbb{Z}_{\geq 0}: \\ a+b \leq n, a-b=d}} \tilde{C}_{n-b}^{a} \cdot\left(\overline{C_{n-a}^{b}} x_{0}^{-1}\right)
$$

for each $n \in \mathbb{Z}_{\geq 0}$ and each $d \in \mathbb{Z}$ with $|d| \leq n$.

Lemma 3.5. Let $d \in[1-n, n-1]$. For each $\mathbf{j}=\left(j_{1}, \ldots, j_{n}\right) \in \mathbf{J}_{n}^{n}$ there exists a unique $a=a(\mathbf{j}, d) \in$ $[\max (0, d), n]$ such that $j_{a} \leq n+d-a \leq j_{a+1}$ (with the convention $j_{0}=0, j_{n+1}=\infty$ ).

Proof. Consider graph of the linear function $y=n+d-x$ on the coordinate plain. Set $a=k$ if there exists $1 \leq k \leq n$ such the point with coordinates $\left(k, j_{k}\right)$ is closest to the graph from the left. Otherwise set $a=0$.

For $a \in[\max (0, d), n]$ denote by $\mathbf{J}_{n}^{n}(a, d)$ the set of all $\mathbf{j} \in \mathbf{J}_{n}^{n}$ such that $a(\mathbf{j}, d)=a$.

We need the following fact (in the notation of Lemmas 3.2 and 3.4).

Lemma 3.6. $\mathbf{J}_{n}^{n}(a, d)=\mathbf{J}_{n+d-a}^{a} \times T^{a}\left(\overline{\mathbf{J}_{n-a}^{a-d}}\right)$.

Proof. Clearly, for any sequence $\mathbf{j} \in \mathbf{J}_{n}^{n}(a, d)$ its subsequence $\mathbf{j}^{\prime}=\left(j_{1}, \ldots, j_{a}\right)$ belongs to $\mathbf{J}_{n+d-a}^{a}$ and the subsequence $\mathbf{j}^{\prime \prime}=\left(j_{a+1}, \ldots, j_{n}\right)$ belongs to $T^{a}\left(\overline{\mathbf{J}_{n-a}^{a-d}}\right)$.

Conversely, it is also clear that for any sequences $\mathbf{j}^{\prime} \in \mathbf{J}_{n+d-a}^{a}$ and $\mathbf{j}^{\prime \prime} \in T^{a}\left(\overline{\mathbf{J}_{n-a}^{a-d}}\right)$ their concatenation $\mathbf{j}=\left(\mathbf{j}^{\prime}, \mathbf{j}^{\prime \prime}\right)$ belongs to $\mathbf{J}_{n}^{n}(a, d)$.

The lemma is proved.

For any two sequences of integers $\mathbf{j}^{\prime}=\left(j_{1}^{\prime}, \ldots, j_{k}^{\prime}\right)$ and $\mathbf{j}^{\prime \prime}=\left(j_{1}^{\prime \prime}, \ldots, j_{\ell}^{\prime \prime}\right)$ define the shifted concatenation by $\mathbf{j}^{\prime} \bullet \mathbf{j}^{\prime \prime}:=\left(\mathbf{j}^{\prime}, T^{k}\left(\mathbf{j}^{\prime \prime}\right)\right)$. We use now an obvious fact that if $\mathbf{j}^{\prime}=\left(j_{1}^{\prime}, \ldots, j_{k}^{\prime}\right), \mathbf{j}^{\prime \prime}=\left(j_{1}^{\prime \prime}, \ldots, j_{\ell}^{\prime \prime}\right)$ then

$$
y_{\mathbf{j}^{\prime} \bullet \mathbf{j}^{\prime \prime}}=y_{\mathbf{j}^{\prime}} y_{\mathbf{j}^{\prime \prime}} .
$$

Then, applying this formula to $\mathbf{j}=\left(\mathbf{j}^{\prime}, T^{a}\left(\mathbf{j}^{\prime \prime}\right)\right) \in \mathbf{J}_{n+d-a}^{a} \times T^{a}\left(\overline{\mathbf{J}_{n-a}^{a-d}}\right)$, we obtain:

$$
\tilde{C}_{n+1}^{n}=\sum_{\mathbf{j} \in \mathbf{J}_{n}^{n}} y_{\mathbf{j}}=\sum_{\substack{a \in[\max (0, d), n], \mathbf{j}^{\prime} \in \mathbf{J}_{n+d-a}^{a}, \mathbf{j}^{\prime \prime} \in \mathbf{J}_{n-a}^{a-d}}} y_{\mathbf{j}^{\prime}} y_{\mathbf{j}^{\prime \prime}}=\sum_{a \in[\max (0, d), n]} \tilde{C}_{n+d-a}^{a} \cdot\left(\overline{C_{n-a}^{a-d}} x_{0}^{-1}\right) .
$$

This proves (3.1).

Theorem 2.22 is proved. 
3.2. Proof of Theorems 2.32 and 2.34. For any set $X$ and $k \geq 0$ denote by $\left\{\begin{array}{l}X \\ k\end{array}\right\}$ the set of all subsets $J \subset X$ of cardinality $|J|=k$. Clearly, $\left\{\begin{array}{c}1, m+n] \\ k\end{array}\right\}=\underset{\substack{a, b \in \mathbb{Z}_{\geq 0}: \\ a+b=k}}{ }\left\{\begin{array}{c}{[1, m]} \\ a\end{array}\right\} \times T^{m}\left(\left\{\begin{array}{c}{[1, n]} \\ b\end{array}\right\}\right)$ for all $m, n, k \in \mathbb{Z}_{\geq 0}$ in the notation of Lemma 3.2, where we view each $J \in\left\{\begin{array}{c}{[1, n]} \\ k\end{array}\right\}$ naturally as an element of $\mathbb{Z}^{b}$.

Taking into account that for $J=\left(J^{\prime}, T^{m}\left(J^{\prime \prime}\right)\right) \in\left\{\begin{array}{c}{[1, m]} \\ a\end{array}\right\} \times T^{m}\left(\left\{\begin{array}{c}{[1, n]} \\ b\end{array}\right\}\right), a+b=k$, we have $y_{J}=$ $T^{m+a}\left(y_{J^{\prime \prime}}\right) y_{J^{\prime}}$ and $y_{J}^{\prime}=T^{b}\left(y_{J^{\prime}}^{\prime}\right) T^{m-a}\left(y_{J^{\prime \prime}}^{\prime}\right)$, we obtain for $m, n, k \in \mathbb{Z}_{\geq 0}$ :

$$
\begin{aligned}
& \begin{array}{c}
\left(\begin{array}{c}
m+n \\
k
\end{array}\right)=\sum_{J \in\left\{\begin{array}{c}
{[1, m+n]} \\
k
\end{array}\right\}} y_{J}=\sum_{J^{\prime} \in\{[1, m]\}, J^{\prime \prime} \in\{[1, n]\}} T^{m-a}\left(y_{J^{\prime \prime}}\right) y_{J^{\prime}}=\sum_{\substack{a, b \in \mathbb{Z} \geq 0: \\
a+b=k}} T^{m+a}\left(\left(\begin{array}{l}
n \\
b
\end{array}\right)\right)\left(\begin{array}{c}
m \\
a
\end{array}\right),
\end{array} \\
& \begin{array}{c}
\left.\left(\begin{array}{c}
m+n \\
k
\end{array}\right)^{\prime}=\sum_{J \in\left\{\begin{array}{c}
{[1, m+n]} \\
k
\end{array}\right\}} y_{J}^{\prime}=\sum_{J^{\prime} \in\left\{\begin{array}{c}
a, b \in \mathbb{Z} \geq 0: a+b=k, \\
1, m] \\
k
\end{array}\right\}, J^{\prime \prime} \in\left\{\begin{array}{c}
{[1, n]} \\
k
\end{array}\right\}} T^{b}\left(y_{J^{\prime}}^{\prime}\right) T^{m-a}\left(y_{J^{\prime \prime}}^{\prime}\right)=\sum_{\substack{a, b \in \mathbb{Z}_{\geq 0}: \\
a+b=k}} T^{b}\left(\left(\begin{array}{c}
m \\
a
\end{array}\right)\right)^{\prime}\right) T^{m-a}\left(\left(\begin{array}{l}
n \\
b
\end{array}\right)^{\prime}\right) .
\end{array}
\end{aligned}
$$

Theorem 2.32 is proved.

Proof of Theorem 2.34 For each $0 \leq j \leq k \leq n$ denote by $\mathbf{I}_{j, k ; n}$ the set of all $\mathbf{i}=\left(i_{1}, \ldots, i_{k}\right) \in \mathbb{Z}_{>1}^{k}$ such that $i_{j} \leq n+k+1-2 j, i_{j+1} \leq n+k-1-2 j, i_{s} \leq i_{s+1}+1$ for all $s \in[1, j]$, and $i_{s}>i_{s+1}+1$ for all $s \in[j+1, k]$. (with the convention that if $j \in\{0, k\}$, then meaningless inequalities are omitted and $\left.\mathbf{I}_{-1, k ; n}=\mathbf{I}_{k+1, k ; n}=\emptyset\right)$.

The following statement is straightforward.

Lemma 3.7. $\tilde{C}_{n+k-j}^{j} \cdot\left(\begin{array}{l}n-j \\ k-j\end{array}\right)=\sum_{\mathbf{i} \in \mathbf{I}_{j, k ; n}} Y_{\mathbf{i}}$ for all $0 \leq j \leq k$, where we abbreviate $Y_{\mathbf{i}}:=y_{i_{1}} \cdots y_{i_{k}}$.

For $j \in[0, k+1]$ denote $\mathbf{I}_{j, k ; n}^{-}=\mathbf{I}_{j-1, k ; n} \cap \mathbf{I}_{j, k ; n}$. By definition, $\mathbf{I}_{0, k}^{-}=\mathbf{I}_{k+1, k}^{-}=\emptyset$ and the following is immediate.

Lemma 3.8. $\mathbf{I}_{j, k ; n}^{-}$is the set of all $\mathbf{i}=\left(i_{1}, \ldots, i_{m}\right) \in \mathbf{I}_{j, k ; n}$ such that $i_{j} \leq i_{j+1}+1$ for all $j \in[0, k]$. In particular, $\mathbf{I}_{j, k ; n}=\mathbf{I}_{j, k ; n}^{-} \sqcup \mathbf{I}_{j+1, k ; n}^{-}$for $j \in[0, k]$.

Using Lemmas 3.7 and 3.8, we obtain for all $0<k \leq n$ :

$$
\sum_{j=0}^{k}(-1)^{j} \tilde{C}_{n+k-j}^{j} \cdot\left(\begin{array}{l}
n-j \\
k-j
\end{array}\right)=\sum_{j \in[0, k], \mathbf{i} \in \mathbf{I}_{j, k ; n}}(-1)^{j} Y_{\mathbf{i}}=\sum_{j \in[0, k], \mathbf{i} \in \mathbf{I}_{j, k ; n}^{-}}(-1)^{j} Y_{\mathbf{i}}+\sum_{j \in[0, k], \mathbf{i} \in \mathbf{I}_{j+1, k ; n}^{-}}(-1)^{j} Y_{\mathbf{i}}=0 .
$$

Theorem 2.34 is proved.

3.3. Proof of Theorems 2.38 and 2.43. We prove Theorem 2.38first. Indeed, the assertion is equivalent to $\left(H_{m}\right)_{i j}=\sum_{k=0}^{\min (i, j)}\left(L_{m}\right)_{i k}\left(U_{m}\right)_{k j}$, i.e., to $C_{m+i+j}=\sum_{k=0}^{\min (i, j)} C_{i+k+m}^{i-k} \cdot x_{2 k+m}^{-1} \overline{C_{k+j+m}^{j-k}}$ for all $i, j \in \mathbb{Z}_{\geq 0}, m \in\{0,1\}$. This identity coincides with that from Theorem 2.22 taken with $n=m+i+j, a=i-k, b=j-k, d=i-j$. Theorem 2.38 is proved.

Proof of Theorem 2.43, It suffices to do so only for $L_{m}^{-}$(the argument for $U_{m}^{-}$is identical). Indeed, the assertion is equivalent to $\sum_{k^{\prime}=i^{\prime}}^{j^{\prime}}\left(L_{m}\right)_{j^{\prime} k^{\prime}}\left(L_{m}^{-}\right)_{k^{\prime} i^{\prime}}=\delta_{i^{\prime} j^{\prime}}$, i.e., to $\sum_{k^{\prime}=i^{\prime}}^{j^{\prime}} \tilde{C}_{j^{\prime}+k^{\prime}+m}^{j^{\prime}-k^{\prime}} \cdot(-1)^{i^{\prime}+k^{\prime}}\left(\begin{array}{c}i^{\prime}+k^{\prime}+m \\ k^{\prime}-i^{\prime}\end{array}\right)=0$ 
for all $0 \leq i^{\prime}<j^{\prime}$. It is easy to show that this identity coincides with that from Theorem 2.34 taken with $n=i^{\prime}+j^{\prime}+m, j=j^{\prime}-k^{\prime} k=j^{\prime}-i^{\prime}$.

Theorem 2.43 is proved.

3.4. Proof of Theorems 2.29, 2.36, We start with a proof of Theorem 2.36, The following is well-known.

Lemma 3.9. Any lower unitriangular $\mathbb{Z}_{\geq 0} \times \mathbb{Z}_{\geq 0}$ matrix $A=\left(a_{i j}\right)$ over an associative unital ring $\mathcal{A}$ is invertible and $\left(A^{-1}\right)_{j i}=\sum_{j=i_{1}>i_{2}>\cdots>i_{k}=i, k \geq 1}(-1)^{k-1} a_{i_{1}, i_{2}} \cdots a_{i_{k-1}, i_{k}}$ for all $1 \leq i \leq j \leq n$.

Applying Lemma 3.9 with $A=L_{m}^{-}$, i.e., $a_{j i}=\tilde{C}_{i+j+m}^{i-j}$ and using Theorem 2.43 in the form $\left(A^{-1}\right)_{j i}=$ $(-1)^{i+j}\left(\begin{array}{c}i+j+m \\ j-i\end{array}\right)$, we obtain the first identity. Swapping $A$ and $A^{-1}$, we obtain the second one.

Theorem 2.36 is proved.

Proof of Theorem 2.29, Recall from [14] that for any matrix over a commutative ring, its determinant equals the product of its principal quasiminors. Let $\underline{H}_{m}^{n}=\chi_{q}\left(H_{m}^{n}\right)=\left(c_{i+j+m}(q, 1)\right), i, j=0, \ldots, n$, where $\chi_{q}: \mathbb{Z} F \rightarrow \mathbb{Z}\left[q, q^{-1}\right]$ is defined in Section 2] Since all principal submatrices of $\underline{H}_{m}^{n}$ are $\underline{H}_{m}^{k}, k=0,1, \ldots, n$, these and Corollary 2.40 guarantee that $\operatorname{det}\left(\underline{H}_{m}^{n}\right)=\prod_{k=0}^{n} \chi_{q}\left(x_{m+2 k}\right)=q^{\sum_{k=0}^{m} \frac{(m+2 k)(m+2 k-1)}{2}}=q^{\frac{n(n+1)(4 n-1+6 m)}{6}}$.

Theorem 2.29 is proved.

\section{REFERENCES}

[1] M. Aigner, Catalan-like numbers and determinants, Journal of Combinatorial Theory, Series A, vol. 87, (1999), pp. 33-51.

[2] G. Andrews, Catalan numbers, q-Catalan numbers and hypergeometric series, Journal of Combinatorial Theory, Series A, vol. 44 (1987), pp. 267-273.

[3] A. Berenstein, V. Retakh, Noncommutative marked surfaces, Advances in Math., vol. 328 (2018), pp. $1010-1087$.

[4] A. Berenstein, V. Retakh, A short proof of Kontsevich cluster conjecture, C.R. Acad. Sci., Paris, Ser. I, vol. 349 (2011), pp. 119-122.

[5] A. Berenstein, V. Retakh, C. Reutenauer, D. Zeilberger, The Reciprocal of $\sum_{n \geq 0} a^{n} b^{n}$ for non-commuting $a$ and $b$, Catalan numbers and non-commutative quadratic equations, Contemp. Math., vol. 592 (2013), pp. 103-109.

[6] L. Carlitz, Sequences, paths, ballot numbers, Fibonacci Quart., vol. 10 (1972), pp. 531-549.

[7] L. Carlitz, J. Riordan, Two element lattice permutation numbers and their $q$-generalization, Duke Math. J., vol 31 (1964), pp. 371-388.

[8] Ph. Di Francesco, R. Kedem, Noncommutative integrability, paths and quasideterminants, Advances in Math., vol. 228 (2011), pp. 97-152.

[9] J Fürlinger, J Hofbauer, q-Catalan numbers, Journal of Combinatorial Theory, Series A, vol. 40 (1985), pp. 248-264.

[10] A. Garsia, M. Haiman, A Remarkable $q$, $t$-Catalan Sequence and $q$-Lagrange Inversion, Journal of Algebraic Combinatorics 1996, vol. 5 (1996), pp. 191-244.

[11] A. Garsia, J. Haglund, A proof of the $q$, t-Catalan positivity conjecture, Discrete Mathematics vol. 256 (2002), pp. $677-717$.

[12] I. Gelfand, D. Krob, A. Lascoux, B. Leclerc, V. Retakh, J.-Y. Thibon, Noncommutative symmetric functions, Advances in Math. vol. 112 (1995), pp. 218-348.

[13] I. Gelfand, S. Gelfand, V. Retakh and R. Wilson, Quasideterminants, Advances in Math., vol. 193 (2005), pp. 56-141.

[14] I. Gelfand, V. Retakh, Determinants of matrices over noncommutative rings, Funct. Anal. Appl. 25 (1991), no. 2, pp. 91102.

[15] I. Gelfand, V. Retakh, A theory of noncommutative determinants and characteristic functions of graphs, Funct. Anal. Appl. vol. 26 (1992), no. 4, pp. 1-20.

[16] J. Haglund, The $q$, t-Catalan Numbers and the Space of Diagonal Harmonics, University Lecture Series, vol. 41 (American Mathematical Society, Providence, 2008). With an appendix on the combinatorics of Macdonald polynomials.

[17] I. Macdonald, Symmetric functions and Hall polynomials Second edition. With contributions by A. Zelevinsky. Oxford Mathematical Monographs. Oxford Science Publications. The Clarendon Press, Oxford University Press, New York, 1995.

[18] I. Pak, A. Postnikov, V. Retakh, Noncommutative Lagrange theorem and inversion polynomials, preprint, https://math.mit.edu/ apost/papers/noncom.pdf .

[19] R. Stanley, Catalan numbers, Cambridge University Press, New York, 2015.

[20] U. Tamm, Some aspects of Hankel matrices in coding theory and combinatorics, Electron. J. Combin. vol. 8 (2001), no. 1.

Department of Mathematics, University of Oregon, Eugene, OR 97403, USA

E-mail address: arkadiy@math.uoregon.edu

Department of Mathematics, Rutgers University, Piscataway, NJ 08854, USA

E-mail address: vretakh@math.rutgers.edu 